\title{
RELATO DE UMA EXPERIÊNCIA EM DIVULGAÇÃO E POPULARIZAÇÃO DE CIÊNCIA
}

\author{
Germano Amaral Monerat ${ }^{1}$ \\ Eduardo Vasquez Corrêa Silva ${ }^{2}$ \\ Luiz Gonzaga Ferreira Filho ${ }^{3}$ \\ Enia Mara de Carvalho ${ }^{4}$
}

\begin{abstract}
RESUMO: O projeto de Extensão "Espaço Ciência \& Tecnologia" do Departamento de Matemática, Física e Computação da Universidade do Estado do Rio de Janeiro (UERJ), em desenvolvimento desde 2010, reúne um conjunto de atividades extensionistas voltadas para divulgação e popularização de ciência, visando estimular o gosto por ela em seu público-alvo (segmentado em alunos universitários da área de ciências exatas, alunos e professores de Física e Matemática do ensino médio e alunos do ensino fundamental). As atividades aqui relatadas tratam de palestras, minicursos e visitas técnicas aos laboratórios de Física da UERJ e oficinas nessa área do conhecimento.
\end{abstract}

PALAVRAS-CHAVE: Extensão universitária. Divulgação científica. Popularização de ciência.

\section{Report on an experiment in science dissemination and popularization}

ABSTRACT: The extension project "Espaço Ciência \& Tecnologia" of the Department of Mathematics, Physics and Computation of the State University of Rio de Janeiro (State of Rio de Janeiro, Brazil), which has being developed since 2010, gathers a set of extension activities oriented to the dissemination and the popularization of science aiming to stimulate the taste for science in its target audience (constituted by exact science university students, high school Physics and Mathematics teachers, and elementary students). The activities reported in this text consist of seminars, short courses, technical visits to the Physics laboratoies of the university, and Physics workshops.

KEYWORDS: University extension. Scientific dissemination. Science popularization.

\section{INTRODUÇÃO}

Longe de ter caráter definitivo e imutável, o conceito e a prática da ação extensionista na universidade brasileira sofreu mudanças ao longo do tempo, o que pode ser atestado pelos documentos legais, que regulamentaram ou regulamentam a educação nacional.

\footnotetext{
${ }^{1}$ Doutor em Física pela Universidade Federal Fluminense, professor adjunto da Universidade do Estado do Rio de Janeiro (monerat@uerj.br).

${ }^{2}$ Doutor em Física pelo Centro Brasileiro de Pesquisas Físicas, professor associado da Universidade do Estado do Rio de Janeiro (eduardo.vasquez@pq.cnpq.br).

${ }^{3}$ Doutor em Física pela Universidade de São Paulo, professor associado da Universidade do Estado do Rio de Janeiro (kph120@gmail.com).

${ }^{4}$ Doutora em Agronomia pela Universidade Federal de Lavras, professora do Centro Universitário de Barra Mansa (eniacarvalho@yahoo.com.br).
} 
A extensão universitária surgiu no Brasil entre os anos de 1911 e 1917, por meio de conferências abertas ao grande público, na Universidade Livre de São Paulo, porém com temas não relacionados aos problemas sociais e políticos da época (CARBONARI; PEREIRA, 2007). De acordo com Duch (2006), o conceito de extensão não surgiu juntamente com o de universidade, que nasceu, primeiramente, com a função do ensino (transmissão de conhecimentos técnico-científicos) e, mais tardiamente, abrigou atividades de pesquisa (produção de tais conhecimentos). Somente a partir da década de 1930, a extensão (difusão e popularização de conhecimentos) começou a delinear-se como atribuição da Universidade.

Em 1930, foi criado, por meio do Decreto $n^{0}$ 19.402/1930, o Ministério dos Negócios da Educação e Saúde Pública e, posteriormente, estabelecido o Estatuto das Universidades Brasileiras (Decreto no 19.851/1931). Esse último sinaliza, pela primeira vez, a existência legal da atividade de extensão.

Um exemplo concreto de ação extensionista desse período é o da Escola Nacional de Agronomia (criada pelo Decreto $\mathrm{n}^{\mathrm{o}} 23.857 / 1934$ ), que teve ações específicas de difusão de conhecimentos científicos em agropecuária para a população rural. Nas décadas seguintes, entretanto, a atividade extensionista perdeu espaço no ambiente universitário. Em 1937, no então Distrito Federal (cidade do Rio de Janeiro), foi criada a Universidade do Brasil, por meio da Lei $\mathrm{n}^{\circ} 452$, reformulando a antiga Universidade do Rio de Janeiro (posteriormente, a partir de 1965, Universidade Federal do Rio de Janeiro), que foi, então, instituída com objetivos claros de ensino e pesquisa, mas tendo a função de extensão omitida de seu estatuto.

Posteriores alterações no Estatuto das Universidades Brasileiras (Decreto-Lei no 8.457/1945) e no Estatuto da Universidade do Brasil (Decreto $n^{\circ}$ 20.445/1946) não mencionam as atividades extensionistas, bem como os de outras universidades federais na década de 1950 . Somente na década de 1960, é que o quadro começa a reverter-se, inserindo-se, novamente, a extensão como atribuição universitária, na Lei de Diretrizes e Bases da Educação Nacional (Lei $\mathrm{n}^{\circ} 4.024 / 1961$ ), característica então propagada para os estatutos das universidades nas décadas de 1960 e 1970. Por exemplo, é explícita a tríplice função de ensino, pesquisa e extensão na criação da Universidade Rural do Rio de Janeiro (Decreto $n^{0}$ 1.984/1963). Apesar dessa reversão, a extensão revestiu-se de caráter assistencialista e prestadora de serviços, e não de aperfeiçoamento social. A Universidade é, então, vista como a depositária de conhecimentos, tendo como função repassá-lo à sociedade.

Segundo Duch (2006), o conceito contemporâneo de extensão começou a esboçar-se com Paulo Freire (1969), elevando-o à comunicação da universidade com sociedade, em uma troca transformadora de conhecimentos. A partir de 1980, a extensão voltaria a ser considerada nas atividades oficiais do magistério superior (Decreto $n^{\circ}$ 85.487/1980). Em 1987, criou-se o Fórum de Pró-reitores de Extensão das Universidades Públicas Brasileiras (FORPROEX), e a extensão passou a integrar os projetos político-pedagógicos das instituições, assumindo caráter interdisciplinar (GARAFA, 1989). O Art. 207 da Constituição Federal de 1988 estabelece que "as universidades gozam de autonomia didático-científica, administrativa e de gestão financeira e patrimonial, e obedecerão ao princípio de indissociabilidade entre ensino, pesquisa e extensão". Em 1996, foram estabelecidos os critérios de avaliação do ensino superior (Decreto $n^{\circ}$ 2.026/1996), incluindo a integração social da Universidade através de seus programas de extensão.

Também a atual Lei de Diretrizes e Bases da Educação Nacional (Decreto $n^{0}$ 9.394/1996) atribui às universidades o papel de "instituições pluridisciplinares de formação dos quadros profissionais de nível superior, de pesquisa, de extensão e de domínio e cultivo do saber 
humano", e o Decreto $n^{\circ} 2.306 / 1997$ regulamenta o regime de trabalho integral como sendo "aquele com obrigação de prestar quarenta horas semanais de trabalho, na mesma instituição, nele reservado o tempo de pelo menos vinte horas semanais destinado a estudos, pesquisa, trabalhos de extensão, planejamento e avaliação". No âmbito da Universidade do Estado do Rio de Janeiro, a Resolução no 002/1998 alterou o nome do Conselho Superior de Ensino e Pesquisa para Conselho Superior de Ensino, Pesquisa e Extensão, além de alterar diversos pontos do Estatuto dessa instituição, de modo a inserir a extensão. Atualmente, as políticas extensionistas das universidades públicas são norteadas pelo Plano Nacional de Extensão (PNE) (BRASIL, 2001).

Em 2012, o FORPROEX (2012) definiu que

a extensão universitária, sob o princípio constitucional da indissociabilidade entre ensino, pesquisa e extensão, é um processo interdisciplinar, educativo, cultural, científico e político que promove a interação transformadora entre Universidade e outros setores da sociedade.

Dessa forma, a atividade de desenvolvimento de projetos extensionistas para divulgação e popularização de ciências se enquadra nos objetivos da extensão universitária ${ }^{5}$, conforme estabelecido pelo PNE.

O processo de difusão e popularização dos conhecimentos técnico-científicos tem pelo menos dois grandes objetivos. O primeiro é o de apresentar, para o grande público, resultados e aplicações de pesquisas científicas, procurando despertar a atenção geral para os imensos benefícios que tais resultados e aplicações trazem ou podem vir a trazer para o cotidiano da população, o que pode atrair o apoio para as atividades investigativas da ciência. A pesquisa tecnológico-científica consiste, em geral, de procedimentos caros, demorados e exigentes de recursos, tanto na formação de mão de obra especializada quanto na aquisição de bens materiais apropriados para sua execução. Levando-se em conta que o financiamento dessas pesquisas é, basicamente, feito pelo Estado, vemos a importância de termos a opinião pública em favor de gastos do orçamento do Estado destinados a essas atividades. O segundo objetivo do processo de difusão e popularização da ciência e da tecnologia é o de estimular as mentes jovens, desde a infância e em todas as camadas sociais, para as carreiras científicas e tecnológicas. É preciso preparar os cidadãos para que sejam participativos, e uma importante parcela dessa capacidade tem como base o desenvolvimento da curiosidade e do senso crítico, mediante o conhecimento científico adquirido na escola e na análise pertinente das informações e atualizações recebidas sobre os avanços da ciência e da tecnologia. Trata-se também de desenvolver a capacidade de inovar e de produzir novos conhecimentos e soluções tecnológicas, adequadas às novas necessidades sociais, ambientais e individuais.

O campus Regional de Resende da UERJ foi criado em 1992, no contexto da política de interiorização dessa Universidade. Essa região é bastante conhecida em todo o estado, devido ao seu grande parque industrial, composto por diversas indústrias do setor automobilístico, químico e siderúrgico, entre outros. O campus acolhe a Faculdade de Tecnologia, que possui, entre os seus departamentos, o Departamento de Matemática, Física e Computação. Em 2005, membros organizaram o evento "Semana comemorativa do ano mundial da física" contou com a presença de palestrantes da UERJ e de diversas outras instituições de ensino e pesquisa, como: Universidade Federal Fluminense (UFF), Centro Brasileiro de Pesquisas

\footnotetext{
${ }^{5}$ Uma visão contemporânea sobre as contribuições da extensão universitária para a sociedade pode ser encontrada em Rodrigues (2013).

${ }^{6}$ Detalhes do evento disponíveis em: <http://www.demac.uerj.br/evento(1)2005.htm>.
} 
Físicas (CBPF), Universidade Federal Rural do Rio de Janeiro (UFRRJ) e Universidade Estadual Paulista Júlio de Mesquita Filho (UNESP). Além disso, contou com 298 inscritos, dentre eles, 265 alunos do ensino fundamental e médio de colégios públicos e privados da região. Além de palestras, outras atividades foram desenvolvidas em oficinas de Física, evidenciando o grande interesse dos jovens pelos vários assuntos tratados e a receptividade que eventos assim teriam, se fossem feitos de forma regular.

Em junho de 2009, foi realizada a "II Semana da Física" da Faculdade de Tecnologia. Os resultados positivos desse projeto nos motivaram a ampliar as atividades, que até então se resumiam a palestras, exibição de filmes com orientação científica e a edição de um jornal eletrônico com artigos de divulgação científica. Ficou evidente a necessidade de criação de um espaço extensionista para o desenvolvimento e a diversificação de novas atividades. Submetemos, então, à Fundação Carlos Chagas Filho de Amparo à Pesquisa do Estado do Rio de Janeiro (FAPERJ), o projeto "Espaço Ciência \& Tecnologia", que, desde setembro de 2010, vem sendo desenvolvido com apoio financeiro da FAPERJ e da Sub-reitoria de Extensão e Cultura da UERJ.

\section{METODOLOGIA}

Para atingirmos o objetivo de promover tais atividades de divulgação com regularidade, o primeiro passo foi o registro, em 2010, na Sub-reitoria de Extensão e Cultura da UERJ do projeto "Espaço Ciência \& Tecnologia". Com o apoio financeiro da FAPERJ, foi construída uma oficina de $120 \mathrm{~m}^{2}$ destinada às atividades extensionistas do projeto, desenvolvendo-se, a seguir, um conjunto de atividades, visando alcançar diferentes públicos, a saber: professores de Matemática e de Física da rede de ensino médio da região; alunos do curso de Engenharia de Produção da UERJ; estudantes do ensino médio e, mais recentemente, a partir de 2014, alunos do ensino fundamental de 5 e 6 anos de idade. Cada uma das atividades promovidas estão descritas abaixo.

\section{Oficinas de Física para professores}

Atualmente, é enorme a carência de instalações e equipamentos para laboratórios nas escolas públicas estaduais de ensino médio. No âmbito específico do ensino de Física, isso acarreta grandes dificuldades no processo de aprendizagem. Equipamentos de laboratórios produzidos comercialmente são, em geral, muito caros, e exigem treinamento do professor, além de manutenção especializada e cara. Por outro lado, equipamentos confeccionados pelo próprio professor, a partir de materiais alternativos, apresentam algumas vantagens: permitem a realização de um grande número de experimentos nos quais, em geral, podem ser atingidos os mesmos objetivos que aqueles realizados com os equipamentos comerciais; são de fácil manutenção e reposição; são muito mais baratos; envolvem o próprio professor, desde a concepção, a quantificação e qualificação do material necessário, o desenho e a montagem até a utilização dentro do laboratório. Como resultado, para além dos aspectos econômicos, esse envolvimento do professor propicia um considerável ganho didático e pedagógico, na medida em que, no processo, ele, certamente, dominará com mais clareza e profundidade os fundamentos a serem demonstrados no decorrer de cada experimento realizado com aqueles equipamentos que ele próprio concebeu e construiu.

Outro ponto importante na motivação para a criação das oficinas é que muitos professores que atuam no ensino médio na região do Médio Paraíba, lecionando as disciplinas de Física e 
Matemática, não possuem formação na área, alguns desses profissionais são formados em áreas bem distintas, como Biologia. Dessa forma, as oficinas constituem uma importante fonte de informação e aperfeiçoamento para as aulas desses profisssionais.

Em 2012, o "Espaço Ciência \& Tecnologia" ofereceu treinamento para doze professores de Física do ensino médio da região, por meio de suas oficinas. Foi realizado um conjunto de experimentos com materiais recicláveis e de baixo custo, que facilita a reprodução nas escolas e permite que os alunos construam seus próprios experimentos. As oficinas foram ministradas em sessões, uma vez a cada quinze dias. Em cada uma, um conjunto de experimentos era discutido e montado juntamente com os professores participantes. Os conceitos teóricos envolvidos eram também discutidos. Cada professor participante, ao término de uma dada oficina, produzia seus próprios experimentos.

Os protótipos foram construídos por bolsistas de extensão. As figuras 1 e 2 exibem dois desses experimentos demonstrativos: o da braquistócrona (trajetória mais rápida) e o de ondas estacionárias, respectivamente. Detalhes referentes a estes e outros experimentos podem ser encontrados no Canal Espaço Ciência Online ${ }^{7}$.

Fig.1- Experimento da braquistócrona.

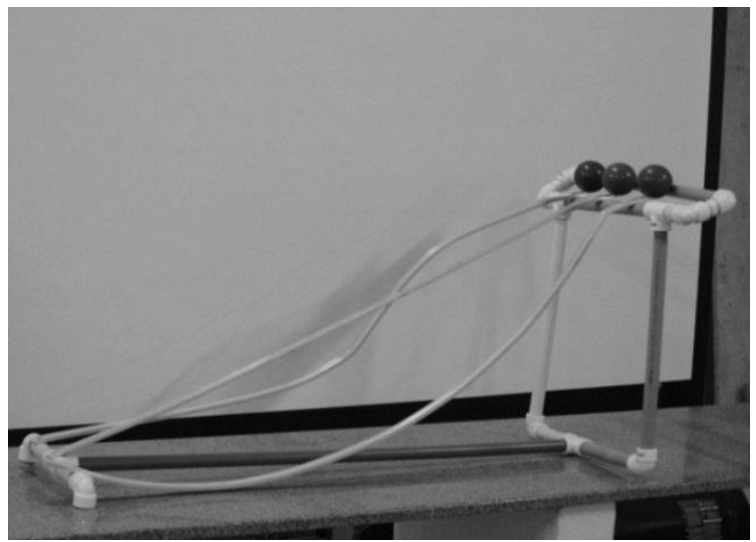

Fonte: Material produzido pelo projeto (2013)
Fig. $2-$ Experimento de ondas
estacionárias.

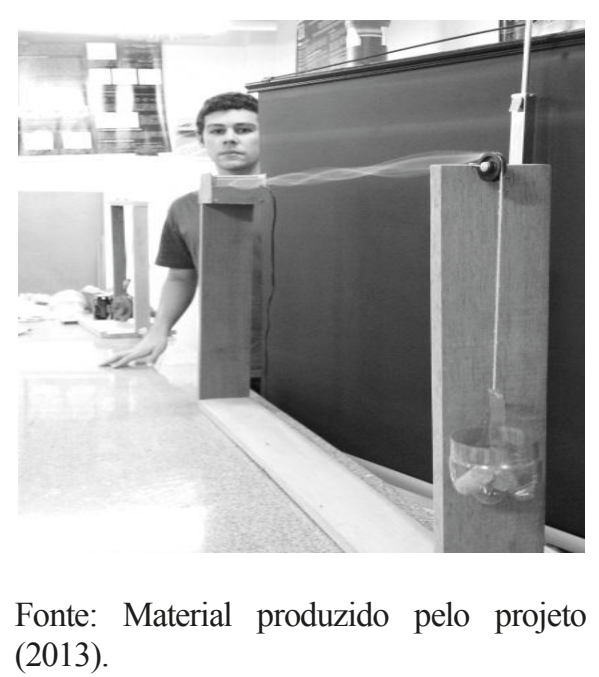

\section{Eventos científicos}

A partir de 2010, o projeto desenvolve quatro eventos científicos com visitas técnicas a laboratórios, palestras e minicursos. As visitas são destinadas exclusivamente para alunos do ensino médio, que são levados aos laboratórios de Física da Faculdade de Tecnologia, onde uma série de experimentos é demonstrada por acadêmicos dos cursos de Engenharia, supervisionados por um docente. Por meio desses experimentos, os conceitos físicos são apresentados as estudantes de forma simples e divertida. $\mathrm{O}$ objetivo é não somente despertar a curiosidade, mas também o gosto pela ciência. As palestras voltadas para divulgação científica são orientadas tanto para acadêmicos da Universidade quanto para estudantes do ensino médio, e algumas delas também são voltadas ao grande público. Os minicursos, por sua vez, são destinados aos alunos dos cursos de Engenharia.

\footnotetext{
${ }^{7}$ Disponível em: <https://www.youtube.com/channel/UCfk1A6OAsGu_er0UOConqpA>. 


\section{Ciência para crianças}

Em 2014, iniciamos a promoção de palestras voltadas para crianças com 5 e 6 anos de idade. Nosso objetivo é aguçar e desenvolver nelas a curiosidade pela ciência. Duas palestras foram apresentadas para alguns grupos com, no máximo, doze crianças. A primeira palestra, "Era uma vez uma menina curiosa" (THOMAZ, 2013a), mostrou a elas como a Matemática está presente em seu cotidiano; como surge a necessidade de se aprender a contar. Em seguida, foi contada como surgiu essa área do conhecimento e, finalmente, discutiu-se o significado das operações matemáticas. Objetivou-se levar para as crianças a mensagem de que, contrariamente a uma visão preconceituosa tão difundida, a Matemática não é tão difícil.

A segunda palestra, intitulada "Era uma vez uma luz que era colorida" (THOMAZ, 2013b), iniciou-se com uma conversa com as crianças a partir de uma história popular que diz que no final do arco-íris existe um pote cheio de ouro, o qual um duende toma conta. Apesar de nunca encontrarmos nem o pote de ouro e nem o duende, a palestrante faz a audiência a indagar sobre a natureza da luz, apresentando de forma simples as ideias de Newton sobre a luz e mostrando como a criança pode criar um arco-íris em dia ensolarado, através da passagem da luz pelas gotinhas de água.

\section{Grande público}

Para que o projeto pudesse atingir o grande público, foi criado um canal no Youtube (ESPAÇO CIÊNCIA, 2010), no qual estão disponibilizados os vídeos das atividades extensionistas (Fig. 3). Atualmente, o canal contém 59 vídeos. Também foi criada uma página na rede social Facebook (ESPAÇO CIÊNCIA, 2011), para a divulgação das atividades do "Espaço Ciência \& Tecnologia" e também de outros projetos, configurando-se em um espaço para discussões e sugestões. Os vídeos são também distribuídos em DVD em algumas escolas da região, para compor o acervo da videoteca.

Fig. 3 - Layout da página principal do canal Espaço Ciência Online.

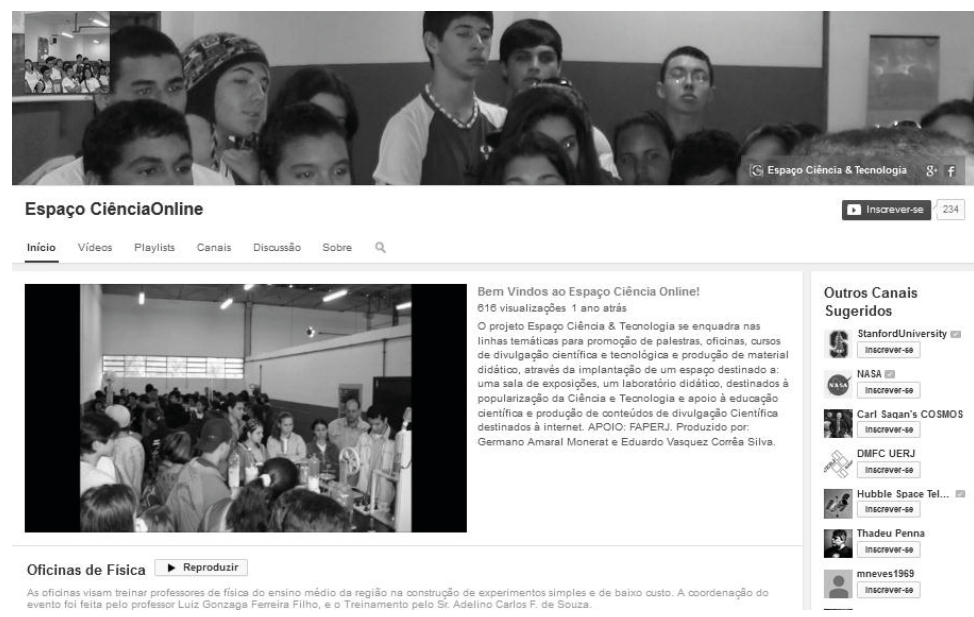

Fonte: Material produzido pelo projeto (2013). 


\section{RESULTADOS}

No que se refere às oficinas de Física para professores do ensino médio, observou-se o efeito multiplicador almejado, por meio de relatos dos participantes. Segundo eles, diversos experimentos foram refeitos em suas respectivas escolas, sendo muitos reproduzidos por seus alunos. Destacamos o trabalho do Prof. Mario Luiz da Silva, que disponibiliza vídeos com diversas atividades no Youtube (LUIZ SILVA, 2011), dentre eles alguns experimentos.

No decorrer de quatro anos, foram realizados quatro eventos: a "III Semana da Física", em 2010; o "I e II Ciclos de Palestras Ciência \& Tecnologia", ambos em 2011; e o "III Ciclo de Palestras Ciência \& Tecnologia", em 2013. Participaram desses eventos quase mil pessoas, entre estudantes e professores do ensino médio e acadêmicos da Faculdade de Tecnologia. Todas as atividades promovidas pelo projeto "Espaço Ciência \& Tecnologia" foram filmadas e seus vídeos editados para divulgação no Canal Espaço Ciência Online e na forma de DVD para distribuição em escolas da região.

Em relação à avaliação do impacto junto ao grande público, é possível aferir o número de acessos a cada vídeo no Canal Espaço Ciência Online e verificar os comentários dos inscritos. Atualmente, o canal possui mais de 250 subscrições e mais de 57.000 acessos, ou seja, aproximadamente 40 acessos por dia. No Facebook, há 153 inscritos e 306 fotos disponibilizadas.

Outro resultado importante do projeto é a utilização de alguns aparatos experimentais produzidos nos laboratórios de física básica nos cursos de Engenharia da Faculdade de Tecnologia da UERJ. Após quatro anos de funcionamento, hoje, há quatro alunos que participam do projeto, sendo três deles voluntários, o que demonstra o crescente interesse pelas atividades de divulgação científica.

\section{AGRADECIMENTOS}

Os autores agradecem à Fundação Carlos Chagas Filho de Amparo à Pesquisa do Estado do Rio de Janeiro (FAPERJ), pelo apoio financeiro (processo $\mathrm{n}^{\circ}$ E-26/112.232/2012).

\section{REFERÊNCIAS}

BRASIL. Ministério da Educação e Cultura. Plano Nacional de Extensão Universitária. Edição Atualizada 2000/2001. Fórum dos Pró-reitores de Extensão das Universidades Públicas. Brasília, MEC, 2001.

CARBONARI, M. E. E.; PEREIRA, A. C. A extensão universitária no Brasil: do assistencialismo à sustentabilidade. Revista de Educação, Valinhos, v. 10, n. 1, 2007. Disponível em: $<$ http://sare.unianhanguera.edu.br/index.php/reduc/article/viewFile/207/205>. Acesso em: 22 abr. 2014. 
DUCH, F. F. Interface extensão universitária e cultura interdisciplinar. 2006. $97 \mathrm{f}$. Dissertação (Mestrado em Semiótica, Tecnologias de Informação e Educação) / Universidade Brás Cubas, Mogi das Cruzes, 2006.

ESPAÇO CIÊNCIA. Página do projeto no canal no Youtube. Resende, RJ: 2010. $<$ https://www.youtube.com/channel/UCfk1A6OAsGu_er0UOConqpA>. Acesso em: 2 ago. 2014.

Página do projeto, na rede social Facebook. Resende, RJ: 2011. $<$ http://www.facebook.com/espaco.ciencia.3>. Acesso em: 2 ago. 2014.

FREIRE, P. Extensão ou comunicação? Rio de Janeiro: Paz e Terra, 1983.

FORPROEX, Fórum de Pró-reitores de Extensão das Universidades Públicas Brasileiras, Política Nacional de Extensão Universitária. Manaus, 2012. Disponível em: $<$ http://www.proec.ufpr.br/downloads/extensao/2012/legislacao/Politica\%20Nacional\%20de \%20Extensao\%20Universitaria\%20maio2012.pdf>. Acesso em: 2 ago. 2014.

GARAFA, V. (Org.) Extensão: a universidade construindo saber e cidadania. Relatório de Atividades 1987/1988. Brasília: Editora da Universidade de Brasília, 1989.

LUIZ SILVA, M. Página pessoal no canal no Youtube. Disponível em: $<\mathrm{http}: / /$ www.youtube.com/user/marioluiz75/featured $>$. Acesso em: 2 ago. 2014.

RODRIGUES, A. L. L. et al. Contribuições da extensão universitária na sociedade. Cadernos de Graduação - Ciências Humanas e Sociais, Aracaju, v. 1, n. 16, p. 141-148, 2013. Disponível em: <https://periodicos.set.edu.br/index.php/cadernohumanas/article/ download/494/254>. Acesso em: 2 ago. 2014.

THOMAZ, M. T. C. dos S. Era uma vez uma menina curiosa. Postado por Espaço Ciência Online. 1 vídeo (29min 58s), son., color. Enviado em 18 set. 2013a. 247 acessos até a presente data. Disponível em: <https://www.youtube.com/watch?v=nKICAH0eeR0>. Acesso em: 2 ago. 2014.

Era uma vez uma luz colorida. Postado por Espaço Ciência Online. 1 vídeo (22min 18s), son., color. Enviado em 18 set. 2013b. 120 acessos até a presente data. Disponível em: $<$ https://www.youtube.com/watch?v=--Ykfc3YMzo>. Acesso em: 2 ago. 2014.

Submetido em 29 de maio de 2014.

Aprovado em 14 de julho de 2014. 